Hospitals NHS Trusts between 2010-2014 were extracted. HIVnegative individuals with $\geq 2$ positive sputum samples or $\geq 1$ positive bronchoalveolar lavage were included. Demographic, clinical, radiological, microbiological, management and outcome data was obtained from electronic records.

Results 1190 NTM sputum samples were identified from 822 individuals. 152 individual patients met inclusion criteria for analysis. Table 1 describes cohort demographics.

Within the cohort 48/152 (32\%) were treated for NTM disease. All treated subjects and 74/104 (71\%) non-treated subjects met international guidelines for diagnosis of NTM infection, which included positive clinical, radiological (cavities or bronchiectasis +/- nodules or infiltrates) and microbiological criteria. Mycobacterium avium complex (MAC) was the most commonly isolated $(68 / 152 ; 45 \%)$ and treated organism $(21 / 48 ; 44 \%)$ followed by Mycobacterium kansasii (11/48; 23\%). 19/48 (40\%) completed treatment (median duration: 17 months [IQR: 1224]). 10/48 (21\%) remain on treatment (median duration: 18 months [IQR: 11-36]), 11/48 (23\%) stopped treatment due to side effects and 13/48 (27\%) were either lost to follow up or treated for Mycobacterium tuberculosis.

Of those treated, 29/48 (60\%) culture converted; 23/29 (79\%) remain negative at 12 months post culture conversion. Of $19 / 48$ who completed treatment, 5/19 (26\%) had symptomatic or radiological disease progression compared to $11 / 28$ (39\%) who did not complete treatment. 11/48 (23\%) patients died within the treatment group. Within the untreated subjects who met international guidelines for NTM infection (74/104), mortality was $19 / 74(26 \%)(\mathrm{p}=0.83)$.

\begin{tabular}{|c|c|c|c|}
\hline Characteristic & Notes & $\begin{array}{l}\text { Treated } \\
(n=48)\end{array}$ & $\begin{array}{l}\text { Non-treated } \\
(n=104)\end{array}$ \\
\hline Age & Median (IQR) & $63(54-76)$ & $70(56-79)$ \\
\hline Sex & Male (\%) & $28(58)$ & $60(58)$ \\
\hline \multicolumn{4}{|l|}{ Ethnicity } \\
\hline & White $(\%)$ & $25(52)$ & $51(49)$ \\
\hline & Black (\%) & $5(10)$ & $10(10)$ \\
\hline & Asian (\%) & $14(29)$ & $21(20)$ \\
\hline & SE Asian (\%) & $0(0)$ & $5(5)$ \\
\hline & Other (\%) & 2 (4) & $8(8)$ \\
\hline & Unknown (\%) & 2 (4) & $9(9)$ \\
\hline Immunosuppression & $\mathrm{n}=(\%)$ & $12(25)$ & $38(37)$ \\
\hline Chronic lung disease & $\mathrm{n}=(\%)$ & $29(60)$ & $42(40)$ \\
\hline Diabetes & $\mathrm{n}=(\%)$ & 2 (4) & $8(8)$ \\
\hline \multicolumn{4}{|l|}{ Symptoms at diagnosis } \\
\hline & Cough (\%) & $39(81)$ & $73(70)$ \\
\hline & Dyspnoea (\%) & $14(29)$ & $28(27)$ \\
\hline & Weight loss (\%) & $19(40)$ & $17(16)$ \\
\hline & Fever (\%) & $6(13)$ & $20(19)$ \\
\hline & Haemoptysis (\%) & $6(13)$ & $17(16)$ \\
\hline & Night sweats (\%) & $10(21)$ & $8(8)$ \\
\hline & Other $(\%)$ & $6(13)$ & $14(14)$ \\
\hline & Asymptomatic (\%) & $0(0)$ & $7(7)$ \\
\hline
\end{tabular}

Discussion NTM is a challenging disease with only 39\% of eligible subjects receiving treatment and a high associated mortality. Furthermore, only $40 \%$ starting treatment completed it and the
$21 \%$ who remain on treatment have been treated for a median duration of 18 months to date. Unlike similar HIV-negative UK cohorts, MAC pulmonary disease is the most prevalent.

\section{Diagnosis and management of pulmonary arterial hypertension}

\section{P265 THE CLINICAL UTILITY OF BIOMARKERS ASSOCIATED WITH INFLAMMATION AND ENDOTHELIAL DYSFUNCTION IN CTEPH}

${ }^{1} \mathrm{C}$ Hadinnapola, ${ }^{1} \mathrm{M}$ Southwood, ${ }^{1} \mathrm{~J}$ Hernandez-Sanchez, ${ }^{1} \mathrm{~K}$ Sheares, ${ }^{1} \mathrm{~S}$ Preston, ${ }^{1} \mathrm{D}$ Jenkins, ${ }^{2} \mathrm{~N}$ Morrell, ${ }^{1} \mathrm{M}$ Toshner, ${ }^{1} \mathrm{~J}$ Pepke-Zaba. ${ }^{1}$ Papworth Hospital, Cambridge, UK; ${ }^{2}$ University of Cambridge, Cambridge, UK

\subsection{6/thoraxjnl-2015-207770.401}

Introduction Chronic thromboembolic pulmonary hypertension (CTEPH) is a rare form of pulmonary hypertension. Inflammation, defective angiogenesis and endothelial dysfunction have been implicated in its pathogenesis. We assessed the prognostic utility of biomarkers, related to these processes, in pulmonary endarterectomy (PEA) assessment.

Methods 80 patients with CTEPH had serum samples taken immediately prior to PEA and a subset $(\mathrm{n}=54)$ also at followup after PEA. 20 healthy volunteers and 20 patients with idiopathic pulmonary arterial hypertension (IPAH) served as controls. Samples were processed on a custom-designed Luminex multiplex array. Biomarker levels were correlated to haemodynamics and functional assessments. Material removed during PEA and explanted lungs of CTEPH and IPAH patients were additionally analysed using immunostaining.

Results Compared to healthy controls Pre PEA samples showed increases in interleukin (IL)-8, -10, tumour necrosis factor $\alpha$ $(\mathrm{TNF} \alpha)$, high sensitivity C-reactive protein (hsCRP) and angiopoietin 2 (Ang2). Vascular endothelial growth factor (VEGFc) was higher in healthy controls.

Following PEA (6.00 \pm 1.83 months), improvements in haemodynamics and six-minute walk distance were observed compared to baseline (Table 1). Additionally, there were decreases in Ang2 and Endoglin.

Preoperative Ang2 levels were independently associated with baseline pulmonary vascular resistance (PVR) with multiple linear regression $(p<0.0001)$. A similar association was found in IPAH subjects $(\mathrm{p}<0.05)$.

Ang2 expression was demonstrated in the endothelium of distal pulmonary arteries in both IPAH and CTEPH notably in areas of small vessel vasculopathy and in neovessels found in the PEA specimens.

The clinical utility in predicting small vessel vasculopathy and residual CTEPH post-PEA surgery was assessed using a cross validation approach. Baseline Ang2 was a necessary component of the best multiple linear model for predicting PVR at follow up (along with baseline PVR, WHO class, age and the use of PAH targeted therapy) $\mathrm{r}^{2}=0.39, \mathrm{q}^{2}=0.35$.

Conclusion We found only modest increases in any marker of inflammation in CTEPH, they were not normalised by PEA or correlated to disease severity. By comparison Ang2 correlated with haemodynamics and has utility in predicting postoperative outcomes. 
Poster sessions

Abstract P265 Table 1 Characteristics and biomarker assessment of CTEPH and control subjects

\begin{tabular}{|c|c|c|c|c|c|c|}
\hline & \multicolumn{2}{|c|}{ CTEPH Pre PEA } & \multicolumn{2}{|c|}{ CTEPH Post PEA } & \multicolumn{2}{|c|}{ Healthy controls } \\
\hline & Median & $95 \% \mathrm{Cl}$ & Median & $95 \% \mathrm{Cl}$ & Median & $95 \% \mathrm{Cl}$ \\
\hline Age & 63.59 & $58.6-66.2$ & 64.09 & $60.98-66.59$ & 60.64 & $58.00-61.46$ \\
\hline IL6 (pg/ml) & 0.13 & $0.13-0.13$ & 0.13 & $0.13-0.13$ & 0.13 & $0.13-0.13$ \\
\hline IL8 $(\mathrm{pg} / \mathrm{ml})^{\mathrm{f}}$ & 7.22 & $4.76-9.34$ & 4.12 & $1.84-5.68$ & 0.13 & $0.13-1.94$ \\
\hline IL10 (pg/ml) $)^{\mathrm{fS}}$ & 1.85 & $1.29-2.96$ & 1.44 & $0.91-2.18$ & 0.13 & $0.13-0.20$ \\
\hline $\mathrm{TNF} \alpha(\mathrm{pg} / \mathrm{ml})^{\mathbb{E}}$ & 9.69 & $7.89-11.10$ & 9.21 & $6.46-10.91$ & 4.72 & $0.45-6.03$ \\
\hline hs CRP $(\mathrm{pg} / \mathrm{ml})^{\mathrm{f}}$ & 2.86 & $2.23-3.91$ & & & 0.72 & $0.62-2.01$ \\
\hline VEGFa (pg/ml) & 157.68 & $121.21-244.79$ & 142.37 & $53.71-178.69$ & 36.78 & $13.70-167.42$ \\
\hline VEGFc $(p g / m l)^{\mathrm{f} \$}$ & 21.96 & $8.71-35.73$ & 17.10 & $9.33-25.14$ & 54.22 & $49.25-62.74$ \\
\hline VEGFd (pg/ml) & 30.51 & $6.90-50.36$ & 27.72 & $8.93-38.97$ & 86.18 & $36.16-108.62$ \\
\hline Ang2 $(\mathrm{pg} / \mathrm{ml})^{* \mathrm{f}}$ & 1266.79 & $1079.31-1649.42$ & 757.07 & $545.04-922.97$ & 575.99 & $539.78-642.32$ \\
\hline BMP9 (pg/ml) & 25.47 & $18.13-28.79$ & 24.87 & $18.49-33.77$ & 29.56 & $16.63-40.97$ \\
\hline Endoglin (pg/ml)* & 242.30 & $209.53-285.24$ & 117.22 & $34.50-160.43$ & 61.71 & $27.40-247.32$ \\
\hline ProBNP (pg/ml) & 575.00 & $130.1-1142$ & 234.00 & $195.50-270.00$ & & \\
\hline RAP $(m m H g)$ & 8.00 & $7.00-9.00$ & 7.00 & $6.00-8.00$ & & \\
\hline $\operatorname{mPAP}(\mathrm{mmHg})^{*}$ & 44.50 & $39.50-47.00$ & 26.00 & $24.00-27.00$ & & \\
\hline $\mathrm{Cl}\left(\mathrm{L} / \mathrm{min} / \mathrm{m}^{2}\right)^{*}$ & 2.29 & $2.19-2.40$ & 2.51 & $2.44-2.66$ & & \\
\hline PVR (Wood units)* & 6.40 & $5.52-8.52$ & 2.80 & $2.60-3.06$ & & \\
\hline $6 m w t$ distance $(m)^{*}$ & 267 & $242-316$ & 353 & $328-380$ & & \\
\hline WHO class* & 3 & $3-3$ & 2 & $2-2$ & & \\
\hline
\end{tabular}

*Variables different between Pre and Post PEA samples.

fVariables different between Pre PEA and healthy control samples.

SVariables different between Post PEA and healthy control samples.

IL - interleukin, TNF $\alpha$ - tumour necrosis factor $\alpha$, hsCRP - high sensitivity C-reactive protein, VEGF - vascular endothelial growth factor, Ang2 - angiopoietin 2, BMP9 - bone morphogenetic protein 9, ProBNP - prohormone brain natriuretic peptide, RAP - right atrial pressure, mPAP -mean pulmonary artery pressure, $\mathrm{Cl}$ - cardiac index, PVR - pulmonary vascular resistance, 6 mwt - six minute walk test.

\section{P266 DO ENDOTHELIN-1 AND INFLAMMATION PLAY A ROLE IN AIRWAY OBSTRUCTION IN PULMONARY ARTERIAL HYPERTENSION ASSOCIATED WITH CONGENITAL HEART DISEASE?}

${ }^{1} \mathrm{AT}$ Low, ${ }^{2} \mathrm{~S} J$ George, ${ }^{2} \mathrm{AB}$ Millar, ${ }^{1} \mathrm{RMR}$ Tulloh. ${ }^{1}$ University Hospitals Bristol, Bristol, UK; ${ }^{2}$ University of Bristol, Bristol, UK

10.1136/thoraxjnl-2015-207770.402
Introduction Airway obstruction has been demonstrated in patients with Pulmonary Arterial Hypertension Associated with Congenital Heart Disease (CHD-APAH), but the cause is unknown. The vasoactive mediator endothelin-1 is a potent vasoconstrictor that induces smooth muscle proliferation in pulmonary arterial hypertension. Endothelin-1 also has the potential to cause bronchoconstriction when present in the airways, though this has not been demonstrated in CHD-

Abstract P266 Table 1 Serum and induced sputum cytokine and endothelin-1 levels for CHD-APAH patients, CHD patients and healthy controls

\begin{tabular}{|c|c|c|c|c|c|}
\hline & Analyte (pg/ml) & CHD-APAH & CHD & Healthy control & $p$ value \\
\hline \multirow[t]{7}{*}{ Serum $(n=56)$} & IL1 $\beta$ & $1(0.52-2.4)$ & $0.36(0.22-1.18)$ & $0.43(0.04-0.78)$ & $0.0214^{*}$ \\
\hline & IL6 & $2.70(1.96-3.97)$ & $1.69(1.2-1.88)$ & $1.53(1.02-1.86)$ & $0.0005^{*}$ \\
\hline & IL8 & $12.3(10.5-15.5)$ & $8.62(6.78-15.28)$ & $9.26(6.12-12.18)$ & $0.0161^{*}$ \\
\hline & IL10 & $0.71(0.26-1.01)$ & $0.65(0.54-1.18)$ & $0.47(0.18-0.76)$ & 0.1119 \\
\hline & TNF $\alpha$ & $12.9(10.82-15)$ & $11.97(9.8-14.42)$ & $10.95(7.38-12.36)$ & $0.0411^{\#}$ \\
\hline & VEGF & $78.9(47.7-101.9)$ & $89.6(58.5-115.9)$ & $41.3(27.7-72.0)$ & $0.0232^{\#}$ \\
\hline & ET-1 & $2.43(2.13-3.30)$ & $1.43(1.16-1.72)$ & $1.48(1.20-1.77)$ & $0.0001^{*}$ \\
\hline \multirow[t]{7}{*}{ Sputum $(n=30)$} & IL1 $\beta$ & $18.2(12.2-33.1)$ & $45.4(23.7-61.3)$ & $36.4(22.2-106.2)$ & 0.2126 \\
\hline & IL6 & $10.6(5.4-57.6)$ & $14.7(9.4-35.2)$ & $16.6(6.4-29.2)$ & 0.7159 \\
\hline & IL8 & $712.4(447.1-1246.4)$ & $746.0(620.5-2335.5)$ & $893.4(348.1-2780.1)$ & 0.9351 \\
\hline & IL10 & $0.64(0.46-0.9)$ & $0.74(0.6-0.9)$ & $0.6(0.44-1.5)$ & 0.6519 \\
\hline & TNF $\alpha$ & $5.89(4.56-7.92)$ & $8.36(4.66-17.7)$ & $5.09(3.66-14.84)$ & 0.5719 \\
\hline & VEGF & $323.6(150.6-376.2)$ & $314.5(196.8-464.6)$ & $295.7(255.6-454.4)$ & 0.9645 \\
\hline & ET-1 & $0(0-0.82)$ & $0.56(0-0.82)$ & $0.98(0.55-1.1)$ & 0.1810 \\
\hline
\end{tabular}

Data presented as median (IQR).

$P$ values calculated by Kruskal-Wallis.

*Post hoc comparison showing CHD-APAH levels significantly greater than CHD and significantly greater than healthy controls $(p<0.05)$.

"Post hoc comparison showing CHD-APAH and CHD levels significantly greater than for healthy controls $(p<0.05)$.

$\mathrm{APAH}=$ Associated pulmonary arterial hypertension, $\mathrm{CHD}=$ Congenital heart disease, $\mathrm{ET}=$ endothelin, IL $=$ interleukin, $\mathrm{TNF}=$ tumour necrosis factor, VEGF $=$ vascular endothelial growth factor. 